\title{
PIAS4 is associated with macro/microcephaly in the novel interstitial 19p13.3 microdeletion/ microduplication syndrome
}

Julián Nevado ${ }^{\star 1,2}$, Jill A Rosenfeld ${ }^{3}$, Rocío Mena ${ }^{1}$, María Palomares-Bralo ${ }^{1,2}$, Elena Vallespín ${ }^{1,2}$, María Ángeles Mori ${ }^{1,2}$, Jair A Tenorio ${ }^{1}$, Karen W Gripp ${ }^{4}$, Elizabeth Denenberg ${ }^{4}$, Miguel del Campo ${ }^{5}$, Alberto Plaja ${ }^{5}$, Rubén Martín-Arenas ${ }^{1}$, Fernando Santos-Simarro ${ }^{6}$, Lluis Armengol ${ }^{7}$, Gordon Gowans ${ }^{8}$, María Orera ${ }^{9}$, M Carmen Sanchez-Hombre ${ }^{9}$, Esther Corbacho-Fernández ${ }^{9}$, Alberto Fernández-Jaén ${ }^{10}$, Chad Haldeman-Englert ${ }^{11}$, Sulagna Saitta ${ }^{12}$, Holly Dubbs ${ }^{13}$, Duban B Bénédicte ${ }^{14}, \mathrm{Xia} \mathrm{Li}^{15}$, Lani Devaney ${ }^{16}$, Mary Beth Dinulos ${ }^{17}$, Stephanie Vallee ${ }^{17}$, M Carmen Crespo ${ }^{1}$, Blanca Fernández ${ }^{18}$, Victoria E Fernández-Montaño ${ }^{1}$, Inmaculada Rueda-Arenas ${ }^{1}$, María Luisa de Torres ${ }^{2,18}$, Jay W Ellison ${ }^{19}$, Salmo Raskin ${ }^{20}$, Carlos A Venegas-Vega ${ }^{21,22}$, Fernando Fernández-Ramírez ${ }^{21}$, Alicia Delicado ${ }^{2,18}$, Sixto García-Miñaúr ${ }^{2,6}$ and Pablo Lapunzina ${ }^{2,6}$

Array comparative genomic hybridization (aCGH) is a powerful genetic tool that has enabled the identification of novel imbalances in individuals with intellectual disability (ID), autistic disorders and congenital malformations. Here we report a 'genotype first' approach using aCGH on 13 unrelated patients with $19 \mathrm{p} 13.3$ submicroscopic rearrangement (11 deletions and 2 duplications) and review cases in the literature and in public databases. Shared phenotypic features suggest that these patients represent an interstitial microdeletion/microduplication syndrome at $19 \mathrm{p} 13.3$. Common features consist of abnormal head circumference in most patients (macrocephaly with the deletions and microcephaly with the duplications), ID with developmental delay (DD), hypotonia, speech delay and common dysmorphic features. The phenotype is associated with at least a $\sim 0.113 \mathrm{Mb}$ critical region harboring three strong candidate genes probably associated with DD, ID, speech delay and other dysmorphic features: MAP2K2, ZBTB7A and PIAS4, an E3 ubiquitin ligase involved in the ubiquitin signaling pathways, which we hypothesize for the first time to be associated with head size in humans.

European Journal of Human Genetics (2015) 23, 1615-1626; doi:10.1038/ejhg.2015.51; published online 8 April 2015

\section{INTRODUCTION}

Chromosome 19 has the highest gene density of the human chromosomes. ${ }^{1}$ However, only a few disease-associated microdeletion/duplication regions have been described. ${ }^{2-11}$ On the short arm, microdeletion/duplication syndromes have been proposed for $19 \mathrm{p} 13$. $13,{ }^{2} 19 \mathrm{p} 13.12^{3}$ and terminal $19 \mathrm{p} 13.3$ microdeletions. ${ }^{4}$ Pure terminal 19p13.3 duplications ${ }^{7}$ have also been described as syndromic entities.

High-resolution microarray comparative genomic hybridization $(\mathrm{aCGH})$ is a powerful genetic tool implemented as a first-tier test for diagnosis of genomic imbalances. ${ }^{12,13}$ A 'genotype first' approach, in which patients are characterized by a similar genomic rearrangement before a common clinical presentation is observed, has proven to be successful in characterizing the growing list of microdeletion/ duplication syndromes. In fact, using this experimental approach, we and others recently described new microdeletion or microduplication syndromes. ${ }^{14-17}$

Here we report 13 new patients with proximal 19p13.3 submicroscopic rearrangements (11 deletions and 2 duplications) and review patients from the literature ( 14 cases; 13 deletions and 1 duplication) ${ }^{18-22}$ and public genomic databases such as DECIPHER and ISCA Consortium (10 cases; 6 deletions and 4 duplications) for a total of 37 cases. We describe the phenotypic findings and suggest that these patients represent a new microdeletion/duplication syndrome at $19 \mathrm{p} 13.3$, with a $113.5 \mathrm{~Kb}$ critical region harboring three genes: ZBTB7A, MAP2K2 and PIAS4, the latter being a candidate gene for abnormal head size.

\footnotetext{
${ }^{1}$ Section of Functional and Structural Genomics Instituto de Genética Médica y Molecular (INGEMM)-IdiPAZ, Hospital Universitario La Paz, Madrid, Spain; ${ }^{2}$ CIBERER, Centro de Investigación Biomédica en Red de Enfermedades Raras, ISCIII, Madrid, Spain; ${ }^{3}$ Signature Genomic Laboratories, PerkinEImer Inc., Spokane, WA, USA; ${ }^{4}$ AI DuPont Hospital for Children, Wilmington, DE, USA; ${ }^{5}$ Hospital Vall D'Hebron, Barcelona, Spain; ${ }^{6}$ Section of Clinical Genetics, INGEMM-IdiPAZ, Hospital Universitario La Paz, Madrid, Spain; ${ }^{7}$ QGenomics Laboratory, Barcelona, Spain; ${ }^{8}$ University of Louisville, Kentucky, USA; ${ }^{9}$ CGC-Genetics, Madrid, Spain; ${ }^{10}$ Hospital Quirón de Madrid, Madrid, Spain; ${ }^{11}$ Wake Forest University, Winston-Salem, NC, USA; ${ }^{12}$ Medical Genetics Institute, Cedars Sinai Medical Center, Los Angeles, CA, USA; ${ }^{13}$ The Children's Hospital of Philadelphia, Philadelphia, PA, USA; ${ }^{14}$ Hospital Saint Vincent de Paul, Lille, France; ${ }^{15}$ Ameripath Northeast, Shelton, CT, USA; ${ }^{16}$ Henry Ford Health System, Sterling Heights, Michigan, USA; ${ }^{17}$ Dartmouth-Hitchcock Medical Center, Lebanon, NH, USA; ${ }^{18}$ Section Cytogenetics, INGEMM-IdiPAZ, Hospital Universitario La Paz, Madrid, Spain; ${ }^{19}$ Kaiser Permanente, San Francisco, CA, USA; ${ }^{20}$ Center for Health and Biological Sciences, Pontifícia Universidade Católica do Paraná (PUC-PR), Curitiba, Brazil; ${ }^{21}$ Genetic Unit Hospital General de México, México, México; ${ }^{22}$ School of Medicine. Universidad Autónoma de México, México, México

*Correspondence: Dr J Nevado, Section of Functional and Structural Genomics, Instituto de Genética Médica y Molecular (INGEMM), IdiPAZ; Hospital Universitario La Paz, Paseo de la Castellana 261, Madrid 28046, Spain. Tel: +34 91 7277217; Fax: +34 91 2071040; E-mail: jnevado@salud.madrid.org

Received 12 October 2014; revised 23 December 2014; accepted 17 February 2015; published online 8 April 2015
} 


\section{MATERIALS AND METHODS}

\section{Individuals}

Patients 1-4 were referred for genetic assessment and patients 5-9 and 12-13 for a customized aCGH analysis to the Institute of Medical and Molecular Genetics at the University Hospital La Paz in Madrid (Madrid, Spain). Patients 5, 7 and 9-13 were originally ascertained following referral for clinical aCGH testing to Signature Genomics, Spokane, WA, USA. The clinical investigations were performed according to the guidelines in the Declaration of Helsinki. Informed consent was obtained from all family members and specific permission to publish photographs was obtained. The studies were approved by the IRB of both institutions. All data reporting variants/phenotypes linked to all rearrangements described herein have been submitted to DECIPHER public data base.

\section{aCGH, fluorescence in situ hybridization and parent-of-origin analysis with short tandem repeats}

The aCGH experiments were performed using previously described methods. ${ }^{23-26}$ Details on the used arrays and specific techniques are given in Supplementary Materials. FISH studies ${ }^{27}$ and microsatellites analyses were performed following standard procedures.

\section{Detection of breakpoints and junction fragment analysis with long-} range PCR and Sanger sequencing

To determine the sequence at the breakpoints for some 19p13.3 deletions (patients 1-7, 9, 12 and 13), we designed a different set of primers (available upon request); according to our custom 19p13.3 aCGH results and followed previously described methods. ${ }^{16}$

\section{RESULTS}

\section{Clinical data}

Clinical and phenotypic findings of all 13 individuals are listed in Table 1, and the facial phenotypes of some patients are shown in Figure 1 (patients 1, 3-6 and 13). Table 1 also analyzes the frequency of several phenotypic features, and compares with previously reported cases (reviewed in Table 2 and references ${ }^{18-22}$ ). Among them, developmental delay (DD), abnormal head size, speech delay, intellectual disability (ID), feeding problems, hypotonia and other dysmorphic features were the most prevalent, present in most deletion cases. Individuals with duplications share many of these main phenotypic features. Unique traits only reported with microdeletion include ophthalmologic alterations, gastroesophageal reflux, sleep disorders, congenital heart disease, and in a lower incidence, behavioral disorders such as autism or aggressive behavior. Distinct recognizable facial features, including a short philtrum, thin upper lip, abnormal ears, wide nasal bridge, depressed nose and root, downslanting palpebral fissures and hypertelorism were apparent in many patients (see Figure 1 and Tables 1 and 2). Ocular anomalies included strabismus, amblyopia, astigmatism and myopia. Congenital heart disease, such as atrial septal defect (the most frequent), dilated aorta, tetralogy of Fallot, bicuspid aortic valve, prominent aortic root and patent ductus arteriosus were observed. In addition, six out of nine patients, including both individuals with duplication, showed proportionate short stature, and three others had a postnatal growth pattern above the mean. In total, 9/13 showed some height alteration. Interestingly, head circumference was abnormal in all but two patients (patients 6 and 10). Head circumference had an almost complete phenotype-genotype correlation among our and previously reported patients: 21/24 patients with deletion have macrocephaly and 2/3 patients with duplication have microcephaly. In fact, macrocephaly (OFC $>97$ th centile) or microcephaly (OFC $<3$ rd centile) was observed at birth in patients with 19p13.3 genomic rearrangements and remained a medical concern throughout their lives.
Additional cases (without full clinical information available) with genomic rearrangements at this region and published in public databases, such as DECIPHER and ISCA consortium, were summarized in Supplementary data (Supplementary Table S1).

\section{aCGH studies}

Genomic rearrangements within 19p13.3 were demonstrated by aCGH in 13 patients (see Supplementary Figure S1 and Table 3 for genomic coordinates in hg19; NCBI build 37). Eleven had deletions, ranging in size from $151 \mathrm{~kb}$ (patient 11) to $1.70 \mathrm{Mb}$ (patient 7), and two had duplications, $1.48 \mathrm{Mb}$ (patient 10) and $2.39 \mathrm{Mb}$ (patient 8). In all but three (patients 6, 9 and 13), the 19p13.3 deletion was the sole significant aberration (Table 3). In patient 6 we also observed a de novo $0.226 \mathrm{Mb}$ deletion at $19 \mathrm{q} 13.2$, and patients 9 and 13 showed two genomic rearrangements within $19 \mathrm{p} 13.3$ separated by a normal region (see supplementary data Supplementary Figure S3). FISH and/or other aCGH assays confirmed the initial aCGH results (data not shown). Most of the deletions/duplications have different breakpoints, although the breakpoints in some cases are in close proximity (Figure 2 and Supplementary Figure S1 and Table 3). When parental samples were available, the rearrangements were found to be de novo (by means of either FISH or aCGH, see supplementary data).

Among individuals with interstitial 19p13.3 rearrangements (sharing characteristic features of abnormal head size, DD, speech delay, hypotonia and dysmorphic features) the shortest region of overlap (SRO) in our series is delineated distally and proximally by patient 11 with estimated breakpoints at genomic positions chr19:39795684131259; hg19; NCBI build 37 ( $\sim 150 \mathrm{~kb}$; Figure $3 \mathrm{a})$. This SRO is shared by 11 of 13 patients (excluding patient 6 partially and patient 10 totally, both of whom have normal head size) and by 13/14 patients in previously reported cases. ${ }^{18-22}$ This segment of $\sim 150 \mathrm{~kb}$ includes one microRNA (SNORD37) and four RefSeq genes: PIAS4, ZBTB7A, $M A P 2 K 2$ and partially EEF2. Review of additional cases included in the literature and public databases such as DECIPHER and ISCA Consortium (with full clinical data) allowed us to narrow this SRO to a $113.5 \mathrm{~kb}$ segment (chr19:3979568-4093035; hg19; NCBI build 37) and excluded EEF2 (Figure 2). Thus, 31/37 individuals shared this SRO and others overlapped it partially (case 4 in reference, ${ }^{22}$ patient 6 in our series and DECIPHER case 271675; Figure 2). On the other hand, three cases did not share the SRO (patient 10 in our series and DECIPHER cases 259222, 255689), although they shared some clinical findings, such as ID, wide nasal bridge, narrow forehead or vesicoureteral reflux, with the SRO's patients.

\section{Characterization of mechanisms underlying interstitial 19p13.3 genomic rearrangements}

We initially designed a custom aCGH with a significant coverage of 19p13.3 (chr19:1477536-6653608, hg19). Further, long-range PCR and automated sequencing were performed to precisely define the breakpoints in some cases (patients 1-7 and 12, Figure 3; patients 9 and 13, data not shown), followed by bioinformatic analysis in both breakpoints through several web tools showing that those lay next to highly homologous repetitive sequences of SINE or LINE elements, which could mediate those rearrangements directly. Results of repeat element analyses, breakpoints and sequences involved are summarized in Table 4, and showed in detail in supplementary data (Supplementary Figure S2). In addition, we also showed analysis of known repeat and genomic architectural elements, such as palindromic DNA or stem-loop structures within the deleted breakpoints that may also modulate in those non-recurrent genomic rearrangements (Supplementary Tables S2 and S3). 


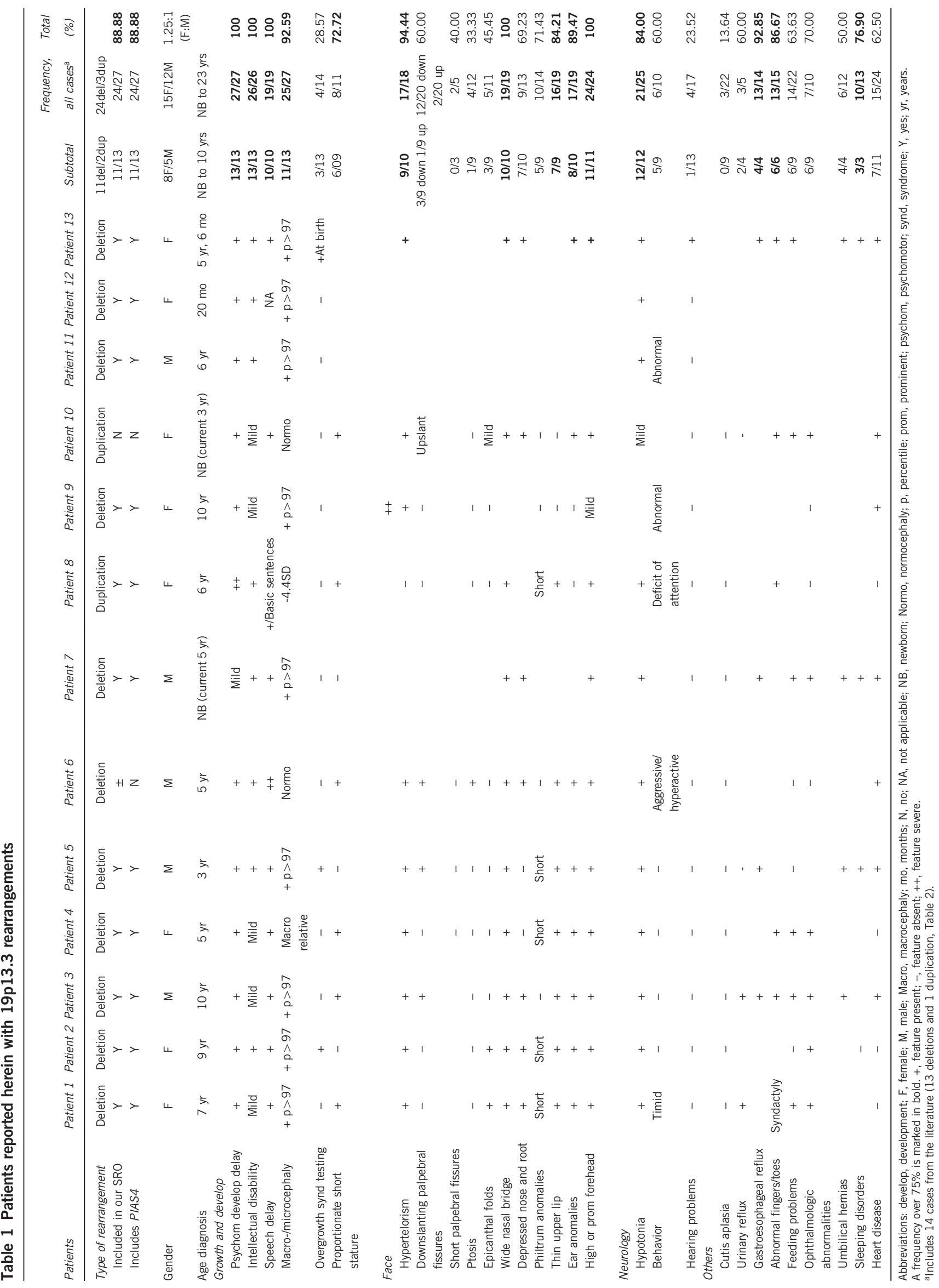




\section{DISCUSSION}

We delineate, review and refine recently described overlapping interstitial deletions/duplications within proximal 19p13.3 (genomic coordinates: 2329320-4996928; genome assembly hg19, NCBI build 37), showing a consistent genotype-phenotype correlation of 13 novel patients, using a 'genotype first approach'. Thus, we propose a novel interstitial microdeletion/duplication syndrome at 19p13.3, centromeric to the $19 \mathrm{p} 13.3$ terminal microdeletions and microduplications. ${ }^{6,7}$

Both deletions and duplications of this region result in some common, non-specific features present in many other microdeletion/ duplication syndromes. Available clinical data presented here, together with cases in the literature ${ }^{8-22}$ and databases, demonstrate several consistent phenotypic findings for this interstitial 19p13.3 deletion: macrocephaly, typically combined with prominent forehead and bitemporal narrowing; facial dysmorphic features such as hypertelorism, depressed nasal bridge and nasal root, short philtrum, thin upper lip and ear anomalies; and developmental and speech delay and ID. Similarly, all patients with duplications of the same region consistently showed microcephaly, dysmorphic facial features (wide nasal bridge, depressed nasal root and hypertelorism), feeding problems in infancy, DD and ID, although dysmorphic features and DD/ID were less severe than in the reciprocal deletions.

\section{Genomic context, deletion size and genes implicated}

The SRO for most cases (31 out of 37) presented here is a $113.5 \mathrm{~kb}$ region harboring three genes: PIAS4, ZBTB7A and MAP2K2 (Figure 2). Although patient 6 (who partially shared the SRO), patient 10 and DECIPHER cases 255689 and 259222 did not share the SRO, they were included in this study because they share many clinical features with patients with deletion or duplication of the SRO. The three SRO genes are involved in diverse functions, including transcription, histone deacetylation and gene translation; ZBTB7A (zinc finger and BTB domain containing 7A) is a transcriptional repressor belonging to the $\mathrm{POK}$ (POZ/BTB) family involved in adipogenesis. $Z B T B 7 A$ may have an important role in neuronal development; ${ }^{28}$ thus deletion of ZBTB7A might contribute to the DD in our patients. In addition, other similar members of this protein family, such as ZBTB20, ZBTB38 and ZBTB18, are strong candidates for the DD in a new microdeletion syndrome at $3 \mathrm{q}^{1} 3^{17}$ and have been associated with human height in GWAS studies ${ }^{29}$ or with several features in 1q43-q44 microdeletion syndrome, ${ }^{30}$ respectively. Dominant gain-of-function mutations of MAP2K2 (mitogen-activated protein kinase kinase 2) cause cardiofaciocutaneous syndrome, ${ }^{31}$ and recently, haploinsufficiency of MAP2K2 has been suggested as a new model of RASopathy in a series of seven patients with deletions. ${ }^{22}$ Finally, PIAS4 (protein inhibitor of activated STAT4) encodes a RING finger (RNF) protein, which interacts with the androgen receptor (AR). PIAS4 is also an E3 ligase involved in ubiquitin signaling pathways. ${ }^{32}$ Additional cases (showing almost the same phenotype) that either partially overlapped or did not include this $113.5 \mathrm{~kb}$ SRO region suggest that part of the phenotypic features could be explained by a 'position effect' or by additional genes close to this SRO (Figure 2). Therefore we hypothesize that the interval from TLE2 to CREB3L3 within 19p13.3, a highly conserved region among different species $^{33,34}$ with a rich content in haploinsufficient genes (with 16/55 with a high likelihood of being haploinsufficient), ${ }^{35}$ as a critical region responsible for most clinical features. Additional genes that may contribute to clinical features are (from centromere to telomere): EEF2, DAPK3, NMRK2, ATCAY and NFIC, among others (Figure 2), which are involved in cognitive impairment ${ }^{36}$ regulation of myogenic differentiation, ${ }^{37}$ apoptosis and transcriptional regulation of canonical $\mathrm{Wnt} / \beta$-catenin signaling, ${ }^{38}$ and autosomal recessive Cayman cerebrallar ataxia, ${ }^{39}$ respectively. NFIC is a member of the nuclear factor I (NFI) gene family necessary for optimal cellular gene expression, ${ }^{40}$ similar to
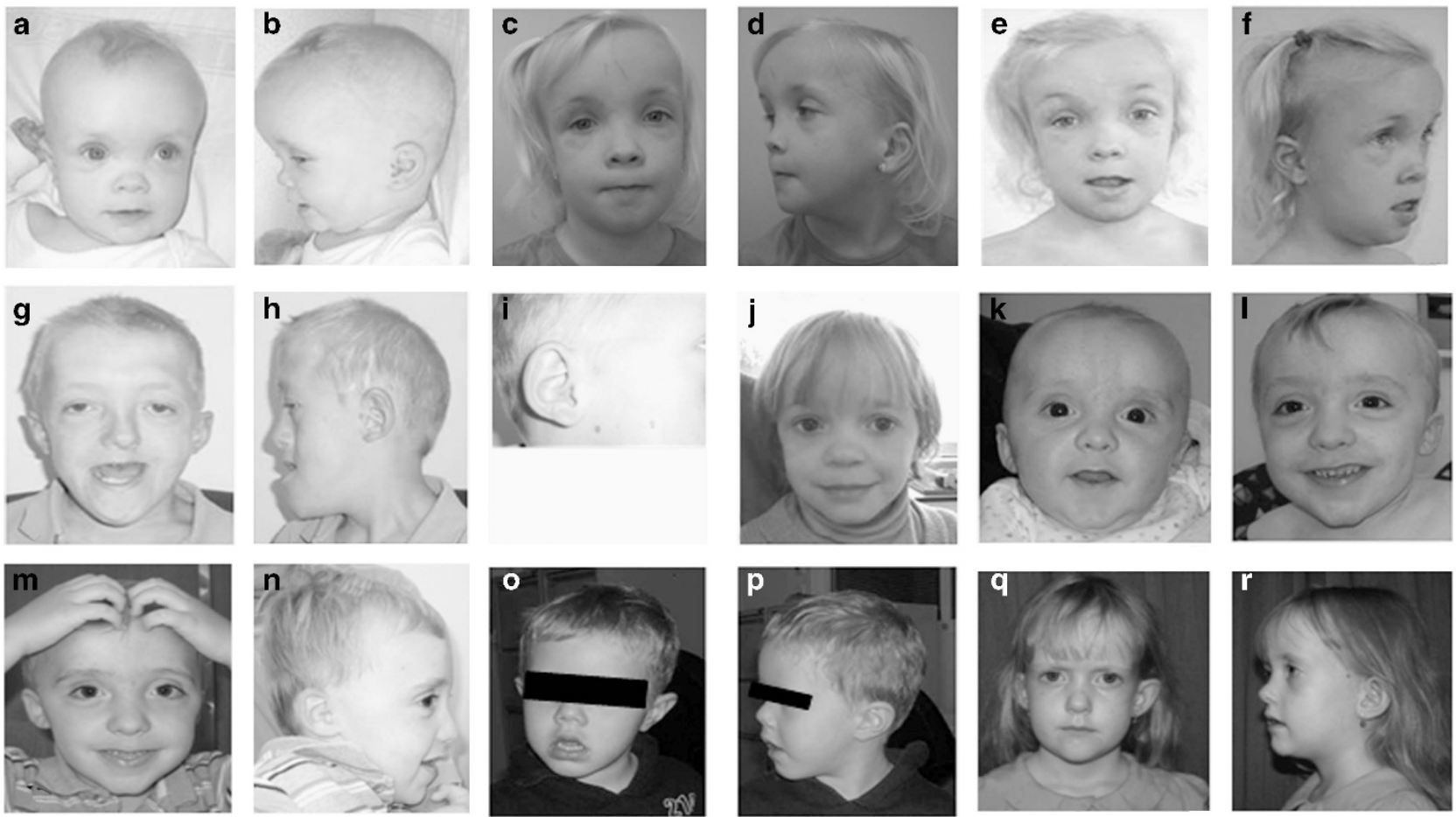

Figure 1 Facial photographs of individuals presented in this study. Patient 1 at age 8 months (a, b), 4 years (c, d) and 7 years (e, f); patient 3 at age of diagnosis, 10 yrs $(\mathbf{g}, \mathbf{h}, \mathbf{i})$; patient 4 at age 5 years $(\mathbf{j})$; patient 5 at age 7 months $(\mathbf{k}), 2$ years $(\mathbf{I})$ and 4 years $(\mathbf{m}, \mathbf{n})$; patient 6 at age 5 years $(\mathbf{o}, \mathbf{p})$; patient 13 at age 8 years $(\mathbf{q}, \mathbf{r})$. 


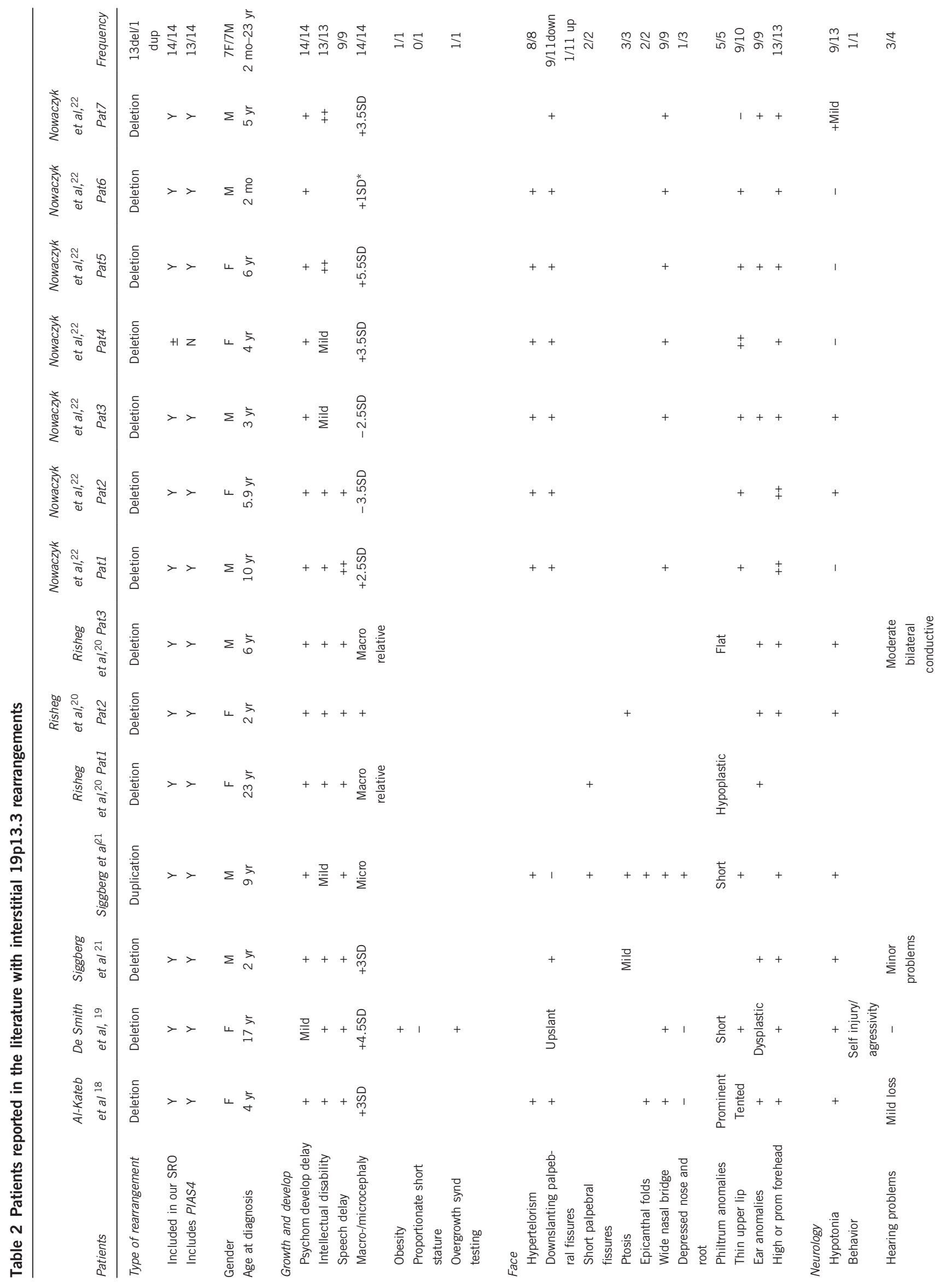




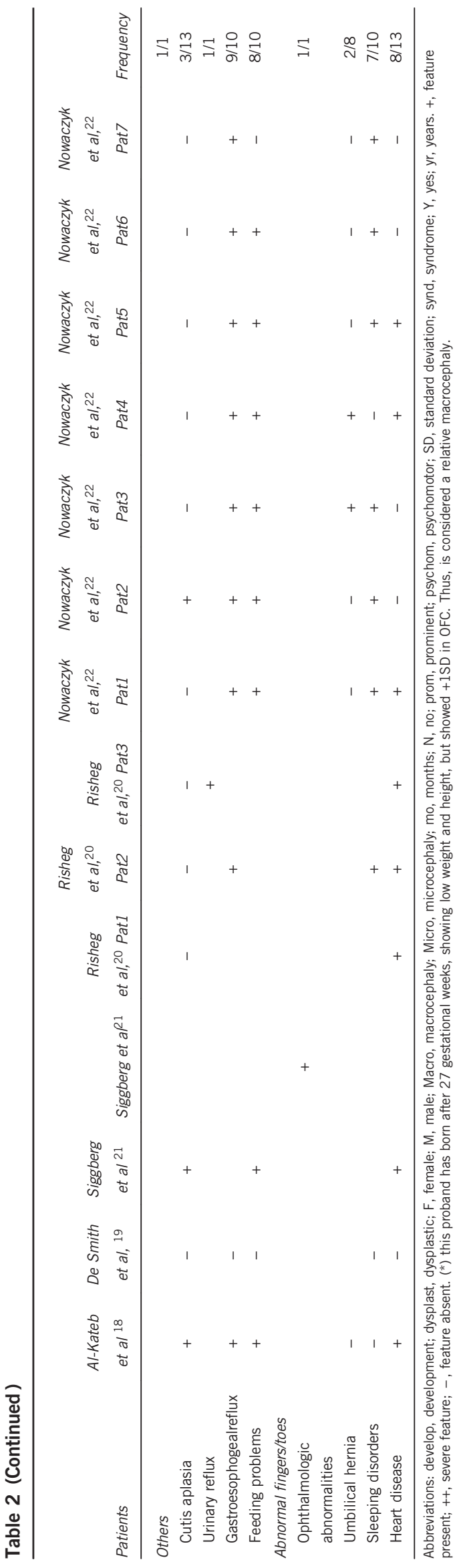

NFIX, a strong candidate for many phenotypic findings similar to those reported here in the recently described 19p13.13 microdeletion/ duplication syndrome (located $\sim 9 \mathrm{Mb}$ centromeric to our SRO). ${ }^{2}$ NFIX has been also implicated in the autosomal dominant Marshall-Smith syndrome (MIM 602535) and in patients with Sotos-like syndrome (currently known as Sotos syndrome type 2; MIM 614753). ${ }^{10}$ Interestingly, deletion of NFIC is observed in 22/37 of patients discussed here (Figure 2). Altogether, these genes appear to be involved in important pathways that could contribute to developmental abnormalities. Additional cases will be needed to refine genes directly implicated in the complete phenotype.

The potential relevance of this $\sim 1.1 \mathrm{Mb}$ interval from TLE2 to CREB3L3 (that includes the SRO) is supported by its highly conserved nature in all vertebrates, from fish to mammals. ${ }^{33,34}$ Further support underlining the importance of this highly conserved region and its contribution to the clinical features in patients with 19p13.3 deletion/duplications is given by their haploinsuffiency (HI) score. HI score (defined by Huang and colleagues, 2010) $)^{35}$ ranges from 0 to 100 , where 0 means highly haploinsufficient and 100 not haploinsufficient. The region from TLE2 to CREB3L3 includes $16 / 55$ potential $\mathrm{HI}$ genes with a score $<50 .{ }^{35}$

\section{Chromosomal architecture context and mechanisms of rearrangement}

The deletions and duplications at $19 \mathrm{p} 13.3$ in patients reported here were not flanked by segmental duplications and mostly unique to each patient, though some are in close proximity (see Figure 2, Table 3). This fact combined with the absence of low copy repeats within or flanking deleted/duplicated regions makes non-allelic homologous recombination (NAHR) an unlikely causative mechanism. Several mechanisms have been proposed for the formation of copy number alterations with non-recurrent breakpoints, and in most microhomology at breakpoint junctions have been invoked. They can be grouped as non-replicative (NAHR and others such as non-homologous end joining (NHEJ) and microhomology-mediated end joining (MMEJ)) or replicative-based repair mechanisms (fork stalling and template switching (FoSTeS), serial replication slippage (SRS), break-induced SRS and microhomology-mediated break-induced replication). ${ }^{41-44}$

We hypothesize that most interstitial non-recurrent $19 \mathrm{p} 13.3$ rearrangements are a consequence of NHEJ and/or the alternative pathway MMEJ, although different patterns have been depicted. Indeed, although repetitive elements of the same family (SINE; Alu: patients 1, 4 and 5) or different family (DNA MER and LINE; patient 2) are present at both breakpoints, the level of sequence identity is probably too low for Alu/Alu-mediated NAHR. In addition, the 4bp, 3bp, $50 \mathrm{bp}$ or 3 bp of microhomology (patients 1,2, 4 and 5, respectively) and/or some changes of nucleotides at the breakpoints (patients 2 and 5) may point to NHEJ or to MMEJ as mechanisms preferentially involved (Table 4,Supplementary Figure S2). In another group of patients, such as patients 6 and 7, only the distal breakpoint showed a repetitive element directly involved, although microhomology of 5- and $6 \mathrm{bp}$, respectively, and some nucleotide insertions observed at the junctions of these deletions, suggested also a MMEJ mechanism (Table 4, Supplementary Figure S2). Finally, a third group of patients (eg, patient 12) did not show any repetitive element directly involved, but close to them. In fact, breakpoints were flanked by AluJo and L1 (LIMB7) elements and the presence of 3 bp microhomology without any scar may point to NHEJ mechanism.

In silico genomic analysis of the breakpoints of the remaining patients (3, 9 and 13) revealed numerous highly repetitive sequences (SINEs/LINEs, see Table 4) that may be involved in the generation of such events. In fact, chromosome 19 contains 'Alus' in the highest 


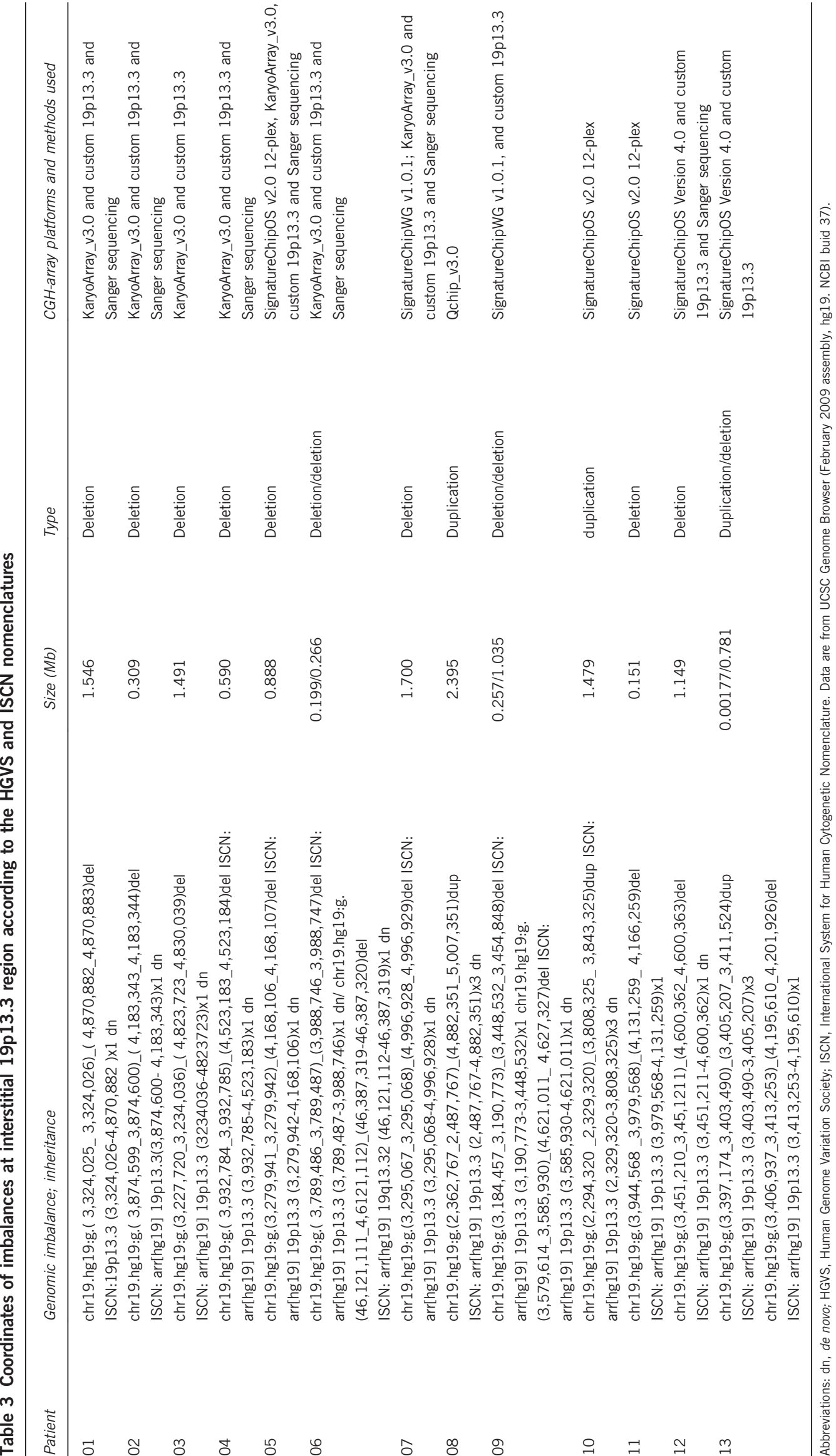




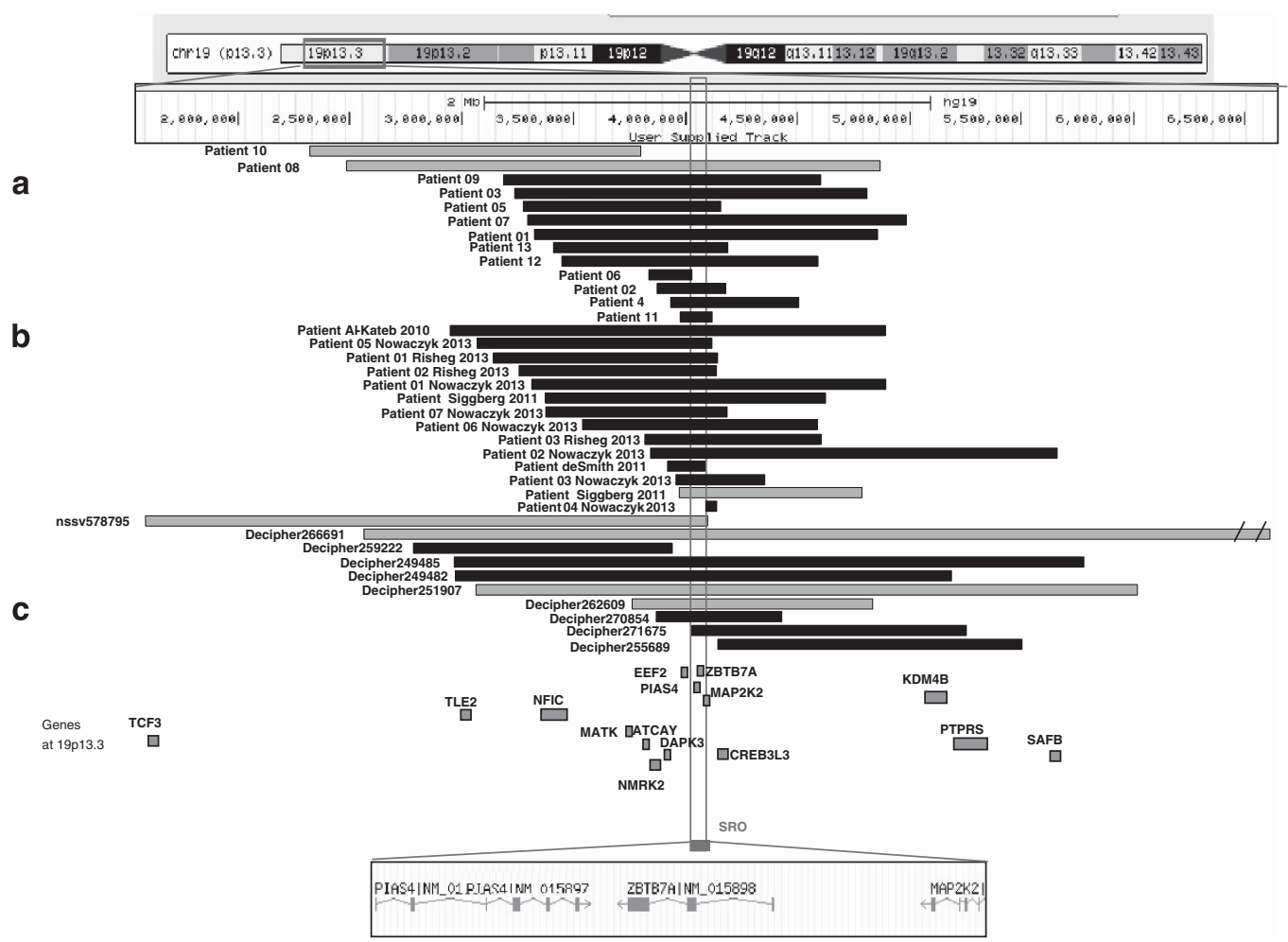

Figure 2 Graphical representation of interstitial 19p13.3 deletions and duplications; genes within the minimal region of overlap. SRO (chr19: 39795684093035; hg19; NCBI build 37). Patients are from our series (a); previous reports (b) and public databases, such as DECIPHER and ISCA consortium (c). Gray; duplications, Black; deletions.

density, comprising $27.2 \%$ of chromosome. ${ }^{45}$ In patients 9 and 13 a replicative mechanism may be suggested by the complex genomic rearrangement observed (deletion-normality-deletion and duplicationnormality-deletion, respectively; see Supplementary Figure S3). Additional cases must be analyzed for a more complete understanding of the mechanisms of deletion and whether genomic architecture in this region predisposes to genomic rearrangements, as previously suggested in other chromosomal regions. ${ }^{46}$ In fact, additional analysis of sequence elements at both breakpoints revealed repetitive elements in all cases, sequences forming nonB-DNA conformations and sequence motifs that may lead to genomic instability and subsequently genomic rearrangements by promoting double strand breaks (DSBs) or by stalling the replication fork as we discussed for the FOXL2 locus $^{47}$ (also see Supplementary Tables S2).

Clinical findings and genotype correlation with head circumference Macrocephaly can be caused by disruption of a broad spectrum of genes and biological functions. There are a few microdeletion/ duplication syndromes with macrocephaly listed in the DECIPHER database: the 1q21.1 microduplication, 8p23.1 duplication, 19p13.13 deletion and Sotos syndrome. In contrast, microcephaly is listed in 16 syndromes in the DECIPHER database. We add the interstitial 19p13.3 microdeletion/duplication syndrome to the differential diagnosis of macrocephaly or microcephaly, respectively. A similar phenomenon of 'mirror' head size phenotypes has been previously reported with genomic rearrangements such as the $19 \mathrm{p} 13.13$ microdeletion/duplication syndrome, ${ }^{2}$ the 1q21.1 microdeletion/duplication syndrome, ${ }^{48}$ and the Sotos syndrome/5q35 duplication syndrome. ${ }^{49}$
A comparative analysis of almost all deleted and duplicated patients pointed to PIAS4 as a strong candidate for abnormal head size (see Figure 3, dashed line), as all patients but one with abnormal head size had deletion or duplication of this gene. In agreement with this, injection of high doses of PIAS into early frog embryos led to reduced head structures with a general and reduced embryonic body length. ${ }^{34}$ In addition, most patients without rearrangements of PIAS4 are normocephalic (Figure 3 panel b). Further support for PIAS genes' implication in head size in humans was obtained reviewing our own database for macrocephalic patients. Indeed, we found a patient with a 3.68 Mb 7p13-p12.3 deletion (43 511 010-47 209 011, genome assembly hg19, NCBI build 37), which included ZMIZ2, a PIAS-like family gene $^{50}$ (see Supplementary Figure S4), as well as that other patients with rearrangement at 7p13-p12.3; cases nssv578186 and nssv584525 and DECIPHER 1224, 1223, 1222, 1221, 1220 (deletions) and nssv579045 (duplication) also showed macrocephaly and microcephaly, respectively.

In contrast, other facts do not support PIAS4's role in head size in humans. Heterozygous and homozygous knockout mice for PIAS4 did not show phenotypic changes. ${ }^{51}$ However, no brain weight measures were taken in the study (Dr Grossched's personal communication). In addition, normocephalic patients have PIAS4 deletions or duplications; 11 of 48 patients in this review had a head size that did not correlate with PIAS4 copy number changes (see Figure 3, mostly in normocephalic individuals in panel b; with asterisks). However, some were evaluated using low-resolution arrays (case 4 in reference ${ }^{22}$ and DECIPHER 255689) or have additional (case 3 in reference ${ }^{22}$ ) or very large chromosomal rearrangements that extend toward the centromere (Figure 3 panel b). We speculate that very large deletions and 
a

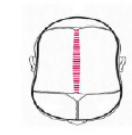

Macrocephaly

b

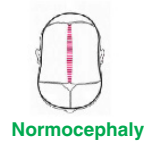

C

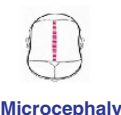

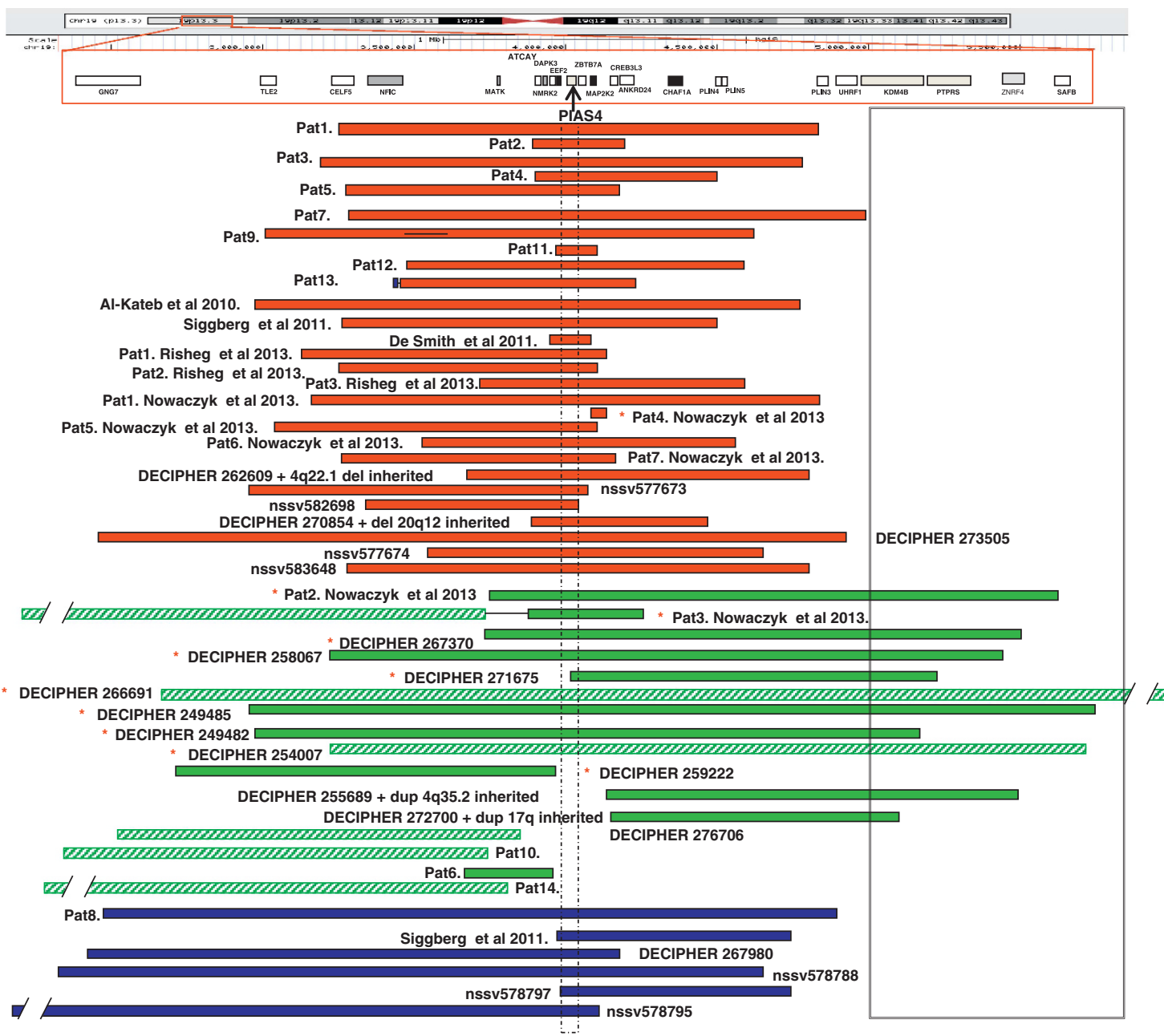

Figure 3 Graphic representation of the minimal region of overlap (SRO, dashed line) responsible for macro/microcephaly in interstitial $19 p 13.3$ microdeletion/duplication syndrome (by means of aCGH). The SRO included only one gene, PIAS4. (a) Almost all patients with macrocephaly (including relative macrocephaly, red bars) have deletions affecting PIAS4. In contrast, all patients with microcephaly (blue bars) have duplications of PIAS4 (c). Patients in panel b, with either deletions (full green bars) or duplications (striped green bars), are normocephalic. The majority of these CNAs either do not include PIAS4 or extend further towards the centromere than the individuals with head size abnormalities. Cases where PIAS4 deletion/duplication status does not correlate with head size (mainly in the normocephalic group) are illustrated by an asterisk $(*)$. The solid black line outlines a putative region which may negate effects of PIAS4 dosage changes. Pat, Patient; nssv, ISCA consortium patients.

duplications extending toward the centromere past genomic coordinate 5000000 (at 19p13.3 band; hg19, NCBI 37) may modify the head size phenotype of PIAS4 dosage change owing to the existence of putative dominant-negative regulator genes within this area. KDM4B, PTPRS and $S A F B$ are predicted haploinsufficient genes ${ }^{35}$ that might be strong candidates for this effect. Mouse knockout studies reveal that these genes are involved in abnormal head morphology $(K d m 4 b)$, decreased brain size by weight (Ptprs), or in smaller fetal size (including head; Safb) compared with littermates (Mouse Genomic Informatic database, MGI; and Mouse Genome Database, MGD; web pages).

The specific mechanism of how PIAS4 or other RNF proteins may be involved in macro/microcephaly is unknown, but PIAS4 is highly conserved from zebrafish to mammals. ${ }^{33,34}$ One hypothesized mechanism is disruption of AR-mediated transcription, which is a common feature of PIAS-like proteins. ${ }^{52}$ A second hypothesis is disruption of bone morphogenetic protein-signaling pathways, as the RING domain of PIAS4 is involved in its suppression. ${ }^{53}$ Third, PIAS4 may be involved in the WNT signaling pathway through its interaction with several transcription factors, ${ }^{32,54}$ and WNT is important for several aspects of facial morphogenesis. Fourth, PIAS4 is an E3 ubiquitin ligase, and haploinsufficiency of E3 ubiquitin ligases in plants and Drosophila melanogaster ${ }^{55-57}$ leads to organ overgrowth. Interestingly, individuals with haploinsufficiency of RNF135 or $R N F 125$, which encode other RNF proteins, have been associated recently (via alteration of ubiquitin signaling pathways) by us and others with macrocephaly and overgrowth in patients with dysmorphic features and ID. ${ }^{58,59}$ Thus, additional efforts will be necessary to definitively establish how PIAS4, PIAS-like or other RNF proteins, may be involved in regulation of human head size.

In summary, after identifying 13 unrelated patients and reviewing others previously reported, we further delineate a novel interstitial microdeletion/duplication syndrome at 19p13.3 (different from subtelomeric microdeletions) whose clinical features overlap many other known microdeletion/duplication syndromes, such as anomalies of head size (macrocephaly in deletions and microcephaly in duplications), pointing to PIAS4 as a putative candidate. This study also provides detailed clinical information for geneticists to assist in the evaluation, diagnosis and management of individuals with similar genomic rearrangements. 


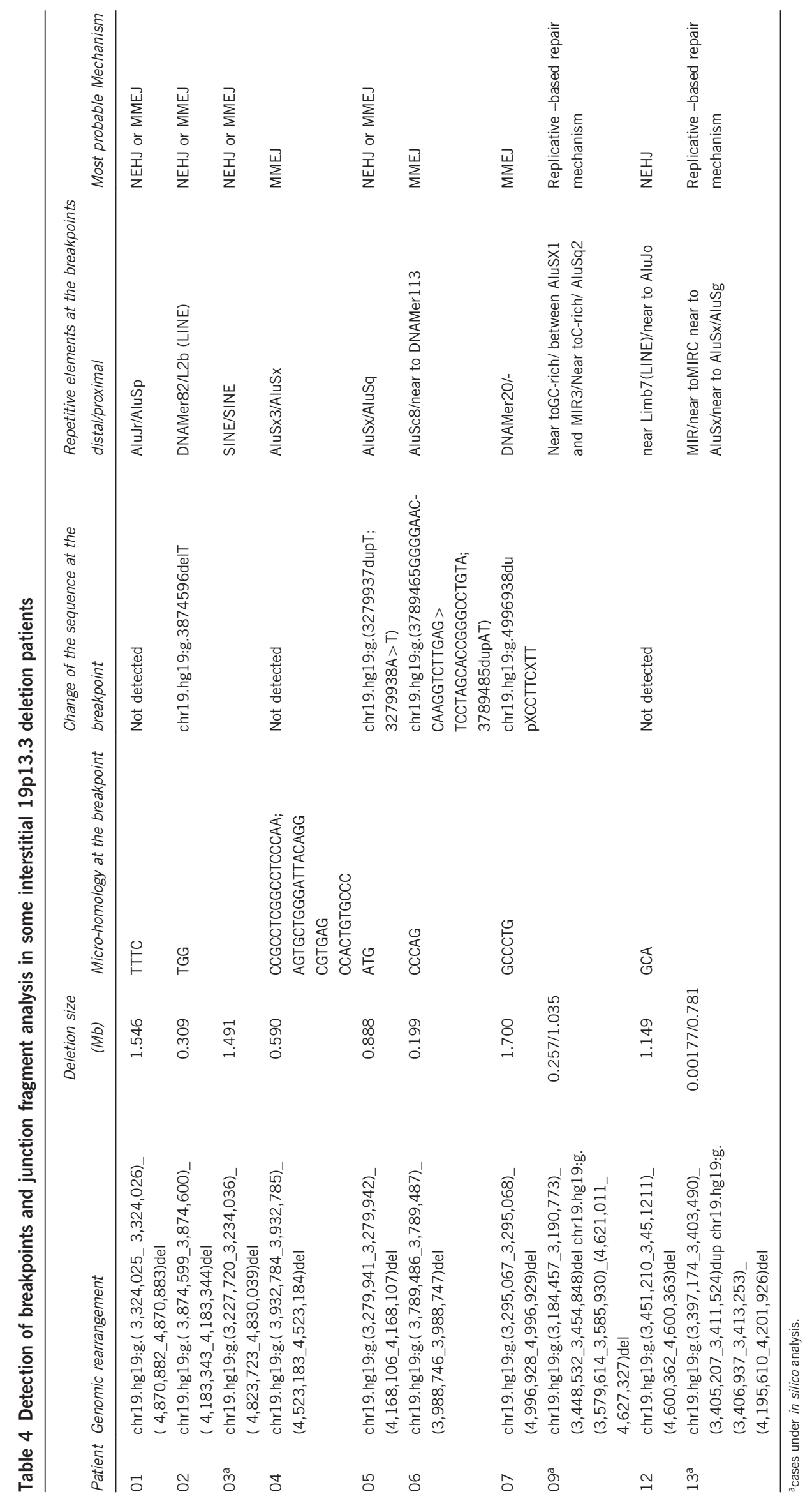




\section{CONFLICT OF INTEREST}

The authors declare no conflict of interest.

\section{ACKNOWLEDGEMENTS}

We would like to thank Drs J Andrieux, N Van der Aa, L Bird, T Cole, K Neas, F Faletra, T de Ravel, N Morrison, four useful and additional information required for several patients in free Databases. This work was supported by a grant from REDES/FIBHULP08 of the Fundación para la Investigación Biomédica Hospital Universitario la Paz, the ENDOSCREEN project from Comunidad Autonóma de Madrid, FIS 011/2491 from ISCIII and the EUCIDCOST Action BM-1208.

\section{AUTHOR CONTRIBUTIONS}

MAM, MPB, EV and JN performed the microarray analysis. JAT did the punctual mutational analysis of PIAS4 gene. MLT, AD and BF did the cytogenetic and FISH studies. IRA and MVFM did Sanger sequencing analysis. IRA and RM did the detection of breakpoints and the junction fragment analysis. RMA and MCC performed the aCGH studies. JN, FSS, SGM, PDL, AD, MC, AP, LD, MO, MCSH, ECF, ASJ, GG, LA, CHE, SS, ED, XL, HD, DBB, SV, MBD, JWE, SR, CAVV, FFR did the clinical characterization of the patients described herein. JAR, KWG, PL and JN wrote the paper in consultation with all the other authors.

1 Grimwood J, Gordon LA, Olsen A et al: The DNA sequence and biology of human chromosome 19. Nature 2004; 428: 529-535.

2 Dolan M, Mendelsohn NJ, Pierpont ME: A novel microdeletion/microduplication syndrome of 19p13.13. Genet Med 2010; 12: 503-511.

3 Kosaki K, Saito H, Kosaki R, Torii C, Kishi K, Takahashi T: Branchial arch defects and 19p13.12 microdeletion: defining the critical region into a $0.8 \mathrm{M}$ base interval. $\mathrm{Am} J$ Med Genet A 2011; 155A: 2212-2214.

4 Archer HL, Gupta S, Enoch S et al: Distinct phenotype associated with a cryptic subtelomeric deletion of 19p13.3-pter. Am J Med Genet A 2005; 136: 38-44.

5 Peddibhotla S, Kaslifa M, Probst FJ et al: Expanding the genotype-phenotype correlation in subtelomeric 19p13.3 microdeletions using resolution clinical chromosomal microarray analysis. Am J Med Genet A 2013; 161A: 2953-2963.

6 Souza J, Faucz F, Sotomaior V, Filho AB, Rosenfeld J, Raskin S: Chromosome 19p13.3 deletion in a child with Peutz-Jeghers syndrome, congenital heart defect, high myopia, learning difficulties and dysmorphic features: Clinical and molecular characterization of a new contiguous gene syndrome. Genet Mol Biol 2011: 34: 557-561.

7 Ishikawa A, Enomoto K, Tominaga M, Saito T, Nagai J, Furuya N, Ueno K, Ueda H, Masuno M, Kurosawa K: Pure duplication of 19p13.3. Am J Med Genet A 2013; 161: 2300-2304.

8 Tentler D, Gustavsson P, Elinder G et al: A microdeletion in 19q13.2 associated with mental retardation, skeletal malformations, and Diamond-Blackfan anaemia suggests a novel contiguous gene syndrome. J Med Genet 2000; 3: 128-131.

9 Zung A, Rienstein S, Rosensaft J, Aviram-Goldring A, Zadik Z: Proximal 19q trisomy: a new syndrome of morbid obesity and mental retardation. Horm Res 2007; 67: 105-110.

10 Malan V, Raoul O, Firth HV: 19q13.11 deletion syndrome: a novel clinically recognisable genetic condition identified by array comparative genomic hybridisation. J Med Genet 2009; 46: 635-640.

11 Chowdhury S, Bandholz AM, Parkash S et al: Phenotypic and molecular characterization of 19q12q13.1 deletions: a report of five patients. Am J Med Genet A 2013; 164A: 62-69.

12 Miller DT, Adam MP, Aradhya S et al: Consensus statement: chromosomal microarray is a first-tier clinical diagnostic test for individuals with developmental disabilities or congenital anomalies. Am J Hum Genet 2010; 86: 749-764.

13 Vermeesch JR, Brady PD, Sanlaville D, Kok K, Hastings RJ: Genome-wide arrays: quality criteria and platforms to be used in routine diagnostics. Hum Mutat 2012; 33: 906-915.

14 Shaffer LG, Theisen A, Bejjani BA: The discovery of microdeletion syndromes in the post-genomic era: review of the methodology and characterization of a new 1q41q42 microdeletion syndrome. Genet Med 2007; 9: 607-616.

15 Mefford HC, Sharp AJ, Baker C et al: Recurrent rearrangements of chromosome 1q21.1 and variable pediatric phenotypes. N Engl J Med 2008; 359: 1685-1699.

16 Palomares M, Delicado A, Mansilla E et al: Characterization of a 8q21.11 microdeletion syndrome associated with intellectual disability and a recognizable phenotype. Am J Hum Genet 2011; 89: 295-301.

17 Molin AM, Andrieux J, Koolen DA et al: A novel microdeletion syndrome at 3q13.31 characterised by developmental delay, postnatal overgrowth, hypoplastic male genitals, and characteristic facial features. J Med Genet 2012; 49: 104-109.

18 Al-Kateb H, Hahn A, Gastier-Foster JM, Jeng L, McCandless SE, Curtis CA: Molecular characterization of a novel, de novo, cryptic interstitial deletion on 19p13.3 in a child with a cutis aplasia and multiple congenital anomalies. Am J Med Genet A 2010; 152A: 3148-3153.
19 de Smith AJ, van Haelst MM, Ellis RJ: Chromosome 19p13.3 deletion in a patient with macrocephaly, obesity, mental retardation, and behavior problems. Am J Med Genet $A$ 2011; 155A: 1192-1195.

20 Risheg H, Pasion R, Sacharow S et al: Clinical comparison of overlapping deletions of 19p13.3. Am J Med Genet A 2013; 161A: 1110-1116.

21 Siggberg L, Olsén P, Näntö-Salonen K, Knuutila S: 19p13.3 aberrations are associated with dysmorphic features and deviant psychomotor development. Cytogenet Genome Res 2011; 132: 8-15.

22 Nowaczyk MJ, Thompson BA, Zeesman S et al: Deletion of MAP2K2/MEK2: a novel mechanism for a RASopathy? Clin Genet 2013; 85: 138-146.

23 Vallespín E, Palomares Bralo M, Mori M et al: Customized high resolution CGH-array for clinical diagnosis reveals additional genomic imbalances in previous well-defined pathological samples. Am J Med Genet A 2013; 161A: 1950-1960.

24 Bejjani BA, Theisen AP, Ballif BC, Shaffer LG: Array-based comparative genomic hybridization in clinical diagnosis. Expert Rev Mol Diagn 2005; 5: 421-429.

25 Duker AL, Ballif BC, Bawle EV et al: Paternally inherited microdeletion at 15q11.2 confirms a significant role for the SNORD116 C/D box snoRNA cluster in Prader-Willi syndrome. Eur J Hum Genet 2010; 18: 1196-1201.

26 Ballif BC, Theisen A, Coppinger J et al: Expanding the clinical phenotype of the 3q29 microdeletion syndrome and characterization of the reciprocal microduplication. $\mathrm{Mol}$ Cytogenet 2008; 1: 8.

27 Traylor RN, Fan Z, Hudson B et al: Microdeletion of 6q16.1 encompassing EPHA7 in a child with mild neurological abnormalities and dysmorphic features: case report. $\mathrm{Mol}$ Cytogenet 2009; 2: 17

28 Artavanis-Tsakonas S, Matsuno K, Fortini ME: Notch signaling. Science 1995; 268 : 225-232.

29 Kim JJ, Park YM, Baik KH et al: Exome sequencing and subsequent association studies identify five amino acid-altering variants influencing human height. Hum Genet 2012; 131: 471-478.

30 de Munnik SA, García-Miñaúr S, Hoischen A et al: A de novo non-sense mutation in ZBTB18 in a patient with features of the 1q43q4 microdeletion syndrome. Eur J Hum Genet 2013; 22: 844-846.

31 Rodriguez-Viciana P, Tetsu O, Tidyman WE: Germline mutations in genes within the MAPK pathway cause cardio-facio-cutaneous syndrome. Science 2006; 311 : 1287-1290.

32 Ihara M, Yamamoto H, Kikuchi A: SUMO-1 modification of PIASy, an E3 ligase, is necessary for PIASy-dependent activation of Tcf-4. Mol Cell Biol 2005; 25 : 3506-3518

33 Burn B, Brown S, Chang C: Regulation of early Xenopus development by the PIAS genes. Dev Dyn 2011; 240: 2120-2126.

34 Xiong R, Nie L, Xiang LX, Shao JZ: Characterization of a PIAS4 homologue from zebrafish: insights into its conserved negative regulatory mechanism in the TRIF, MAVS, and IFN signaling pathways during vertebrate evolution. J Immuno/ 2012; 188: 2653.

35 Huang N, Lee I, Marcotte EM, Hurles ME: Characterising and predicting haploinsufficiency in the human genome. PLoS Genet 2010; 6: e1001154.

36 Verpelli C, Piccoli G, Zibetti C et al: Synaptic activity controls dendritic spine morphology by modulating eEF2-dependent BDNF synthesis. J Neurosci 2010; 30: 5830-5842.

$37 \mathrm{Li} \mathrm{J}$, Rao H, Burkin D, Kaufman SJ, Wu C: The muscle integrin binding protein (MIBP) interacts with alpha7betal integrin and regulates cell adhesion and laminin matrix deposition. Dev Biol 2003; 261: 209-219.

38 Togi S, Ikeda O, Kamitani S et al: Zipper-interacting protein kinase (ZIPK) modulates canonical Wnt/beta-catenin signaling through interaction with Nemo-like kinase and T-cell factor 4 (NLK/TCF4). J Biol Chem 2011; 286: 19170-19177.

39 Bomar JM, Benke PJ, Slattery EL et al: Mutations in a novel gene encoding a CRALTRIO domain cause human Cayman ataxia and ataxia/dystonia in the jittery mouse. Nat Genet 2003; 35: 264-269.

40 Mermod N, O'Neill EA, Kelly TJ, Tjian R: The proline-rich transcriptional activator of CTF/NF-I is distinct from the replication and DNA binding domain. Cell 1989; 58 : 741-753.

41 Hastings PJ, Ira G, Lupski JR: A microhomology-mediated break-induced replication model for the origin of human copy number variation. PLoS Genet 2009; 5: e1000327.

42 Lee JA, Carvalho CM, Lupski JR: A DNA replication mechanism for generating nonrecurrent rearrangements associated with genomic disorders. Cell 2007; 131: 1235-1247.

43 Shaw CJ, Lupski JR: Non-recurrent 17p11.2 deletions are generated by homologous and non-homologous mechanisms. Hum Genet 2005; 116: 1-7.

44 Lieber MR: The mechanism of human nonhomologous DNA end joining. J Biol Chem 2008; 283: 1-5.

45 Tae-Min K, Seung-Jin H, Mun-Gan R: Periodic explosive expansion of human retroelements associated with the evolution of the hominoid primate. J Korean Med Sci 2004: 19: 177-185.

46 Auffray C, Caulfield T, Khoury MJ, Lupski JR, Schwab M, Veenstra T: Looking back at genomic medicine in 2011. Genome Med 2012; 4: 9

47 Verdin H, D'haene B, Beysen D et al: Microhomology-mediated mechanisms underlie non-recurrent disease-causing microdeletions of the foxl2 gene or its regulatory domain. PLoS Genet 2013; 9: e1003358.

48 Brunetti-Pierri N, Berg JS, Scaglia F et al: Recurrent reciprocal 1q21.1 deletions and duplications associated with microcephaly or macrocephaly and developmental and behavioral abnormalities. Nat Genet 2008; 40: 1466-1471.

49 Rosenfeld JA, Kim KH, Angle B et al: Further evidence of contrasting phenotypes caused by reciprocal deletions and duplications: duplication of nsd 1 causes growth retardation and microcephaly. Mol Syndromol 2013; 3: 247-254. 
50 Beliakoff J, Sun Z: Zimp7 and Zimp10, two novel PIAS-like proteins, function as androgen receptor coregulators. Nucl Recept Signal 2006; 4: e017.

51 Roth W, Sustmann C, Kieslinger M: PIASy-deficient mice display modest defects in IFN and Wnt signaling. J Immunol 2004; 173: 6189-6199.

52 Peng Y, Lee J, Zhu C, Sun Z: A novel role for protein inhibitor of activated STAT (PIAS) proteins in modulating the activity of Zimp7, a novel PIAS-like protein, in androgen receptor-mediated transcription. J Biol Chem 2010; 285: 11465-11475.

53 Imoto S, Sugiyama K, Yamamoto T, Matsuda T: The RING domain of PIASy is involved in the suppression of bone morphogenetic protein-signaling pathway. Biochem Biophys Res Commun 2004; 319: 275-282.

54 Sachdev S, Bruhn L, Sieber H, Pichler A, Melchior F, Grosschedl R: PIASy, a nuclear matrix-associated SUMO E3 ligase, represses LEF1 activity by sequestration into nuclear bodies. Genes Dev 2001; 15: 3088-3103.
55 Breuninger $\mathrm{H}$, Lenhard M: Expression of the central growth regulator BIG BROTHER is regulated by multiple cis-elements. BMC Plant Biol 2012; 12: 41.

56 Colombani J, Bianchini L, Layalle S et al: Antagonistic actions of ecdysone and insulins determine final size in Drosophila. Science 2005; 310: 667-670.

57 Gradilla AC, Mansilla A, Ferrús A: Isoform-specific regulation of a steroid hormone nuclear receptor by an E3 ubiquitin ligase in Drosophila melanogaster. Genetics 2011; 189: 871-883

58 Douglas J, Cilliers D, Coleman K et al: Mutations in RNF135, a gene within the NF1 microdeletion region, cause phenotypic abnormalities including overgrowth. Nat Genet 2007: 39: 963-965.

59 Tenorio J, Mansilla A, Valencia M et al: A New Overgrowth Syndrome is Due to Mutations in RNF125. Hum Mutat 2014; 35: 1436-1441.

Supplementary Information accompanies this paper on European Journal of Human Genetics website (http://www.nature.com/ejhg) 